\title{
Severe Pancytopenia and Stomatitis Case due to the Treatment with High Dose Methotrexate
}

\author{
Burcin Meryem Atak Tel*, Satilmis Bilgin, Ozge Kurtkulagi, Gizem Kahveci, Recep Aktas, Kamile Kurt, \\ Tuba Taslamacioglu Duman, Gulali Aktas \\ Department of Internal Medicine, Abant Izzet Baysal University Hospital, Bolu, Turkey.
}

\begin{abstract}
Objective: Methotrexate is used to suppress inflammation in many rheumatologic conditions. Here we present an elderly patient who developed serious side effects due to methotrexate. A 78 year old male admitted to emergency department with oral mucosal bleeding, skin rash, decrease in oral nutrition intake and weakness. He had been using 5 milligrams of methotrexate as 2 tablets twice a day for 10 days, after he was diagnosed with psoriatic arthritis 15 days ago. A diagnosis of methotrexate intoxication established with history, physical examination and laboratory analysis, which revealed pancytopenia. His signs and symptoms, as well as pancytopenia were recovered on $6^{\text {th }}$ day of the hospitalization. He had been treated with folinic acid and filgrastim along with supportive care. Although methotrexate treatment and toxicity is well established it is still a clinical challenge that all clinicians must be aware of. In conclusion, methotrexate intoxication is a clinical entity that can lead to serious clinical consequences, and it is essential to diagnose and initiate appropriate treatment without delay to prevent morbidity and mortality.
\end{abstract}

Keywords: Methotrexate toxicity, Pancytopenia, Treatment, Folinic acid, Filgrastim, Inflammation, Psoriatic arthritis.

\section{INTRODUCTION}

Methotrexate is used in the treatment of pustular, erythrodermic forms and psoriatic arthritis as well as severe, treatment-resistant, moderate to severe plaque psoriasis, and other rheumatologic conditions [1].

As a folic acid analog, methotrexate, binds to dihydrofolate reductase and decreases thymidylate, purine synthesis and cell proliferation [2]. At the same time, it decreases adenosine accumulation and lymphocyte proliferation, production of interleukin- 1 , interferon- $\gamma$ and tumor necrosis factor- $\alpha$, and other inflammatory cytokines, by inhibiting other folate-dependent enzymes such as 5-aminoimidazole-4 carboxamide ribonucleotide transformylase, thus, it has an anti-inflammatory effect by suppressing adhesion and chemotaxis of the neutrophils. In addition, it suppresses keratinocyte proliferation and dermal inflammatory infiltration by decreasing interleukin-22 levels $[2,3]$. The effectiveness of the drug increases in psoriatic arthritis especially when it is combined with biological agents or phototherapy. In this case report, we aimed to present an elderly patient with psoriasis who developed methotrexate related toxicity.

\section{CASE REPORT}

A 78-year-old male patient diagnosed with known psoriasis and benign prostatic hyperplasia who was diagnosed with psoriatic arthritis and methotrexate was started 15 days ago at an external medical center. The patient did not come to the control appointments for a while, however, continued to use 5 milligrams of methotrexate as 2 tablets twice a day for 10

*Address correspondence to this author at the Department of Internal Medicine, Abant Izzet Baysal University Hospital, Bolu, Turkey.

Email: burcinatak@hotmail.com days, due to the worsening itching. Afterwards, he showed up to the emergency clinic of our institution with complaint of oral mucosal bleeding, rashes, decrease in oral nutrition and weakness. In the physical examination of the patient, fever was 36.8 Celsius degree, blood pressures were $120 \mathrm{mmHg}$ systolic and $60 \mathrm{mmHg}$ diastolic, saturation was $96 \%$, and heart rate was 90 beats per minute. Oral examination revealed diffuse oral hemorrhagic lesions and there were palpable purpura overall the skin of the patient (Fig. 1). Rest of the physical examination was unremarkable. Laboratory analyses revealed white blood cell count of $1.5 \mathrm{k} / \mathrm{mm}^{3}$, neutrophil count $0.01 \mathrm{k} / \mathrm{mm}^{3}$, hemoglobin $7.3 \mathrm{~g} / \mathrm{dL}$ and platelet count $4000 / \mathrm{mm}^{3}$. Biochemical tests of the serum were within normal range. According to these findings, a diagnosis of methotrexate intoxication was established and the patient was hospitalized for treatment of pancytopenia, mucositis and skin rash.

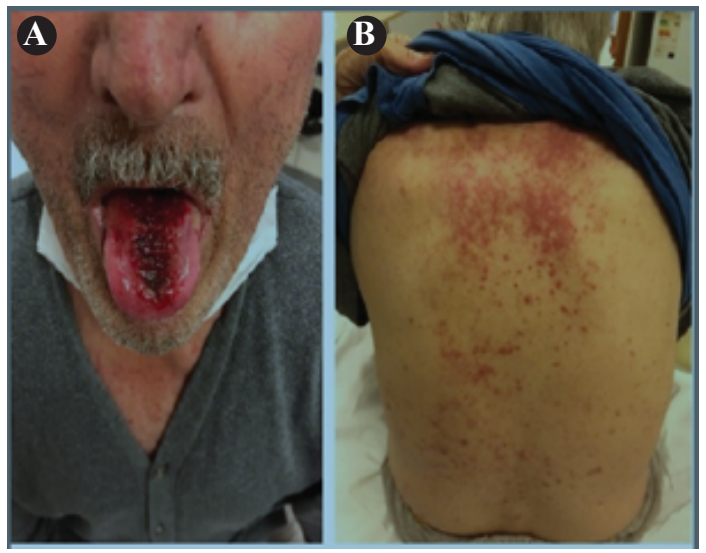

Fig. (1). (A) Oral Hemorrhagic Lesions, and (B) Purpuric Skin Rash on the Back of the Patient.

www.njhsciences.com 
The peripheral smear of the patient was compatible with the current laboratory and no atypical cells were detected. There was no pathological images in the abdominal ultrasound scan and computerized tomography of the thorax. Serological tests for viral pathogens that might suppress the bone marrow were found to be negative. Brucella tube agglutination test was normal. Serum levels of vitamin B12, folate and ferritin were $1254 \mathrm{mg} / \mathrm{dL}, 10 \mathrm{mg} / \mathrm{dL}$ and $2000 \mathrm{mg} / \mathrm{dL}$, respectively. Elevation in serum ferritin was assumed to be an acute phase response.

Because the patient was neutropenic and had a high C-reactive protein level, ceftriaxone and clarithromycin treatment was initiated after an infectious disease consultation. Isotonic and dextrose solutions were infused due to the low oral intake which is caused by painful hemorrhagic oral lesions. Folinic acid infusion of $10 \mathrm{mg} / \mathrm{m}^{2}$ for 3 days was given to reduce the effect of methotrexate. However, because of resistant pancytopenia and mucosal bleeding, the infusion was extended to 6 days with the recommendation of rheumatology department. A 30 million units of filgastrim, granulocyte colony stimulating factor (G-CSF), was administered for 3 days. With the recommendation of dermatology department oral antifungal and antiseptic solutions were given to treat oral lesions. A total of 2 units of pooled platelets and 2 units of erythrocyte suspension were infused during clinical follow up. The pancytopenia improved and oral lesions diminished on the 6th day of the treatment. Repeated hemogram test revealed a white blood cell count of $7.8 \mathrm{k} / \mathrm{mm}^{3}$, with neutrophil count 4.1 $\mathrm{k} / \mathrm{mm}^{3}$. His hemoglobin level was elevated to $9.5 \mathrm{~g} / \mathrm{dL}$ and platelet count was increased to $163000 / \mathrm{mm}^{3}$. The patient was consulted by the department of rheumatology with direct radiographs, and psoriatic arthritis was not considered and his drugs were stopped. General condition of the patient improved and he was discharged with full recovery.

\section{DISCUSSION}

In present case report, we presented a serious Methotrexate toxicity case, which responded well to the treatment.

Methotrexate is a dihydrofolate reductase enzyme inhibitor with very high selectivity. Therefore, it reduces the production of DNA and thymidylate synthesis, and tissues with rapid proliferation. Its effects are particularly prominent in oral mucosa, gastrointestinal system, and bone marrow [2]. The drug should be followed in terms of its side effects as well as its serious efficacy in the disease. Hemogram, liver function tests, kidney function tests, pregnancy test, hepatobiliary ultrasound, chest x-ray, and serum serological tests (HIV, HBV, HCV) should be evaluated prior to initiation of treatment. Administration of $5 \mathrm{mg}$ folic acid 3 days 24 hours after the initiation of methotrexate reduces the risk of drug toxicity. Before the second dose, hemogram, liver and kidney function tests should be examined, especially in elderly patients and patients with renal dysfunction. Methotrexate has low safety threshold and teratogenic effect. Contraception should be recommended for 3 months after drug discontinuation [1].

Measures could be taken to reduce MTX induced side effects including folic acid supplement, symptomatic treatment and careful monitoring. Dose reduction or withdraw of the treatment is necessary if severe side effects happen. In case cytopenia develop as a side effect, the drug dose should be reduced or discontinued completely $[4,5]$. In the presented case, the drug was discontinued and it was not restarted after the treatment completed for toxicity.

Common side effects of methotrexate include nausea, vomiting, stomatitis, elevated liver function tests, headache, weakness, impaired concentration, alopecia, fever, nephrotoxicity, bone marrow suppression, interstitial pneumonia, and alveolitis. Bone marrow suppression and constitutional symptoms were prominent in present case. In multiple cohorts, methotrexate appears to have very few clinically significant side effects, possibly due in part to the routine use of folic acid supplementation [6].

The most important causes of acute methotrexate intoxication are renal dysfunction and overdose of the drug. Aristizabal-Alzate et al. reported a severe case of methotrexate intoxication in the presence of renal impairment which is one of the most common reason of methotrexate intoxication. Like our case, the patient had bone marrow suppression and mucosal lesions. In the presented case, the cause of methotrexate intoxication was long term use of the drug higher than the recommended dose. In case of suspicion of methotrexate intoxication, folinic acid replacement from $10 \mathrm{mg} / \mathrm{m}^{2}$ every 6 hours should be administered as tolerated by the patient $[7,8]$. In accordance with the literature, folinic acid replacement was used in the treatment of the presented case. Aristizabal-Alzate et al. used folinic acid as well as hemodialysis to eliminate the toxic methotrexate. So the therapy should be tailored according to the patient and the present organ dysfunctions [7].

The patient, as mentioned above, developed intoxication due to misuse of methotrexate, and many of the serious side effects of the drug were observed in the clinical course. Interestingly, although severe bone marrow suppression was seen, liver and kidney function tests were not impaired. However, if admission to the hospital was delayed a little longer, it would be probable that prerenal azotemia due to oral intake insufficiency could occurred.

\section{CONCLUSION}

Methotrexate intoxication is a clinical entity that can lead to serious clinical consequences, and it is essential to diagnose and initiate appropriate treatment without delay to prevent 
morbidity and mortality. Clinicians should be well aware about the side-effects of methotrexate and carefully explain the use of methotrexate and the dosage to the patient especially in the elderly patients

\section{CONFLICT OF INTEREST}

Declared none.

\section{ACKNOWLEDGEMENTS}

Declared none.

\section{REFERENCES}

[1) Pathirana D, Ormerod AD, Saiag P, et al. European S3-guidelines on the systemic treatment of psoriasis vulgaris. J Eur Acad Dermatol Venereol 2009; 23(Suppl 2): 1-70.

[2] Kehribar DY, Duran TI, Polat AK, OzgenM. Effectiveness of methotrexate in idiopathic granulomatous mastitis treatment. Am J Med Sci 2020; 360(5): 560-5. DOI: 10.1016/j.amjms.2020.05.029

[3] Strober BE, Menon K. Folate supplementation during methotrexate therapy for patients with psoriasis. J Am Acad Dermatol 2005; 53(4): 652-9. DOI: 10.1016/j.jaad.2005.06.036
[4] Gan EY, Chong WS, Tey HL. Therapeutic strategies in psoriasis patients with psoriatic arthritis: Focus on new agents. BioDrugs 2013; 27(4): 359-73. DOI: $10.1007 / \mathrm{s} 40259-013-0025-6$

[5] Wang W, Zhou H, Liu L. Side effects of methotrexate therapy for rheumatoid arthritis: A systematic review. Eur J Med Chem 2018; 158: 502-16. DOI: 10.1016/j.ejmech.2018.09.027

[6] Yazici Y. Long-term safety of methotrexate in the treatment of rheumatoid arthritis. Clin Exp Rheumatol 2010; 28(5 Suppl 61): S65-S7.

[7] Aristizabal-Alzate A, Nieto-Rios JF, Ocampo-Kohn C, Serna-Higuita LM, Bello-Marquez DC, Zuluaga-Valencia GA. Successful multiple-exchange peritoneal dialysis in a patient with severe hematological toxicity by methotrexate: Case report and literature review. J Bras Nefrol 2019; 41(3): 427-32. DOI: 10.1590/2175-8239-jbn-2018-0095

[8] Nast A, Boehncke WH, Mrowietz U, et al. S3 - Guidelines on the treatment of psoriasis vulgaris (English version). Update. J Dtsch Dermatol Ges. 2012; 10(Suppl 2): S1-95. DOI: 10.1111/j.1610-0387.2012.07919.x 